Research article

Open Access

\title{
The Influence of some Xanthone Derivatives on the Activity of J-774A.1 Cells
}

\author{
Henryk Marona ${ }^{1}$, Elżbieta PęKala ${ }^{*}{ }^{1}$, Agnieszka Gunia ${ }^{1}$, Zenon Czuba ${ }^{2}$, \\ Edward SzNELER ${ }^{3}$, Tadeusz SADOWSKI ${ }^{4}$, Wojciech KRÓL ${ }^{2}$
}

${ }^{1}$ Department of Technology and Biotechnology of Drugs, Jagiellonian University, Medical College, Medyczna 9, 30-680 Cracow, Poland

${ }^{2}$ Department of Microbiology and Immunology, Medical University of Silesia, Jordana 19, 41-808 ZabrzeRokitnica, Poland

${ }^{3}$ Faculty of Chemistry, Jagiellonian University, Ingardena 3, 30-060 Cracow, Poland

${ }^{4}$ Department of Municipal Hygiene, Medical University of Silesia, Medyków 18, 40-752 Katowice, Poland

* Corresponding author. E-mail: epekala@cm-uj.krakow.pl (E. Pękala)

Sci Pharm. 2009; 77: 743-754

doi:10.3797/scipharm.0906-08

Published: October $17^{\text {th }} 2009$

Accepted: $\quad$ October $16^{\text {th }} 2009$

Received: June $9^{\text {th }} 2009$

This article is available from: http://dx.doi.org/10.3797/scipharm.0906-08

(C) Marona et al.; licensee Österreichische Apotheker-Verlagsgesellschaft m. b. H., Vienna, Austria.

This is an Open Access article distributed under the terms of the Creative Commons Attribution License (http://creativecommons.org/licenses/by/3.0/), which permits unrestricted use, distribution, and reproduction in any medium, provided the original work is properly cited.

\begin{abstract}
The chemiluminescence of stimulated cells with phorbol myristate acetate and the production of nitric oxide after stimulation with lipopolisaccharide in the presence of the parent compounds FAA (flavone-8-acetic acid $=(4-0 \times 0-2-$ phenyl-4H-chromen-8-yl)acetic acid), XAA (xanthone-4-acetic acid $=(9-0 \times 0-9 H-$ xanthen-4-yl)acetic acid), and appropriate xanthone derivatives (1-7) was determined. Also the toxicity of the FAA, MFAA ((6-methyl-4-oxo-2-aryl-4Hchromen-8-yl)acetic acid), XAA and 1-7 against J-774A.1 cultured cells was evaluated. Compound 5 (2-methyl-2-\{[(9-oxo-9H-xanthen-2-yl)methyl]sulfanyl\}propanoic acid) was effective in inhibiting chemiluminescence of J-774A.1 cells but most of the other tested compounds stimulated the reaction. FAA and two xanthones with a methoxycarbonyl moeity slightly decreased the generation of nitric oxide at $50 \mu \mathrm{M}$. Most of the tested compounds (1-7) showed weak toxicity at concentration of $100 \mu \mathrm{M}$.
\end{abstract}

\section{Keywords}

Xanthone derivatives $\cdot \mathrm{J}-774 \mathrm{~A} .1$ cells $\bullet$ Chemiluminescence $\bullet$ Toxicity 


\section{Introduction}

Among the different classes of antitumor agents, the some flavone and xanthone derivatives are an important group of compounds with anticancer activity [1,2]. Earlier studies have shown the advantageous properties of synthetic flavone-8-acetic acid (FAA, NSC 347512; (4-oxo-2-phenyl-4H-chromen-8-yl)acetic acid) [3] i.e. against advanced experimental colon tumors in mice [4]. Additionally, FAA has a different toxicity profile to most anticancer drugs, with no significant myelo suppression observed [5]. The related compounds MFAA (6-methyl-4-oxo-2-aryl-4H-chromen-8-yl)acetic acid) containing a 6-methyl substituent in FAA showed antitumor activity comparable to FAA in vitro but were essentially inactive in vivo [6]. Also the closely related class of compounds FAA and MFAA the xanthone-4-acetic acid ((9-oxo-9H-xanthen-4-yl)acetic acid; XAA) [7] and some of its derivatives, in particular 5,6-dimethylxanthone-4-acetic acid ((5,6-dimethyl-9-oxo-9Hxanthen-4-yl)acetic acid; DMXAA) [8,9] showed promising antitumor activity. A recent comparative study of FAA and DMXAA, with a series of derivatives of XAA, in which the substituents in position 5 and 6 were included in five or six-membered rings suggest cytotoxicity activity in a preliminary in vitro assay, comparable to the parent compounds [10].<smiles>O=C(O)Cc1cccc2c(=O)cc(-c3ccccc3)oc12</smiles>

FAA<smiles></smiles>

MFAA<smiles>O=C(O)Cc1cccc2c(=O)c3ccccc3oc12</smiles>

XAA<smiles>Cc1ccc2c(=O)c3cccc(CC(=O)O)c3oc2c1C</smiles>

DMXAA

Sch. 1.

Earlier studies have shown that functions of activated macrophages such as killing of tumor cells, release of cytokines and generation of oxygen radicals can be regulated by flavone [11,12]. They have also been shown to inhibit oxido-reductases [13], thus preventing the formation of free radicals resulting from the reduction of oxygen. FAA and analogues increases the direct cytotoxicity of murine macrophages in vitro against tumor targets [14], and stimulates the formation of nitric oxide [15]. Nitric oxide (NO) is one from a variety of mediators released by activated macrophages. It has been identified as potent molecule that may exert regulatory or cytotoxic effects depending on the concentration acting on the target cell $[16,17]$. The results of our earlier studies [18] suggest that the flavones can modulate the immune responses and the inflammatory reactions by controlling production of nitric oxide. J-774A.1 cells are functional similar to murine macrophages $[19,20]$.

In this study the chemiluminescence of stimulated cells with phorbol myristate acetate (PMA) and the production of nitric oxide after stimulation with lipopolysaccharide (LPS) in the presence of the parent compounds FAA, XAA and xanthone derivatives 1-7 were determined. 


\section{Results and Discussion}

\section{Chemistry}

FAA was derived from the Drug Synthesis and Chemistry Branch, Division of Cancer Treatment, National Cancer Institute, USA, through the courtesy of Dr. Paull. Other xanthone derivatives (XAA and 1-5) were synthesized as previously described [7, 21-23]. Compound 6 was obtained by condensation of 2-methyl-6-hydroxyxanthone [24] with abromopropionic acid according to published procedure for 4 [23]. Compound 7 was readily formylated according to Link [25], using 2-methyl-6-hydroxyxanthone, solid sodium hydroxide, acetone and chloroform. Formation of appropriate analogues of clofibrate under Link conditions is a widely used reaction [26,27]. Some of them (XAA, 2, 6 and 7) were characterized by formation of appropriate esters (XAAa, 2a, 6a and 7a) (Tab.1), according to well known procedures.

Tab. 1. Structure of the synthesized compounds

\begin{tabular}{|c|c|c|}
\hline Cpds & $\mathbf{R}^{1}$ & $\mathbf{R}^{2}$ \\
\hline XAA & $4-\mathrm{CH}_{2}-\mathrm{COOH}$ & $\mathrm{H}$ \\
\hline XAAa & 4-CH $\mathrm{CH}_{2}-\mathrm{COOMe}$ & $\mathrm{H}$ \\
\hline 1 & $2-\mathrm{CH}_{2}-\mathrm{COOH}$ & $\mathrm{H}$ \\
\hline 2 & $2-\mathrm{CH}_{2}-\mathrm{COOH}$ & 6-OMe \\
\hline $2 a$ & 2-CH$-\mathrm{CHOMe}_{2}$ & 6-OMe \\
\hline 3 & $2-\mathrm{CH}_{2}-\mathrm{COOH}$ & $7-\mathrm{Cl}$ \\
\hline 4 & & $\mathrm{H}$ \\
\hline 5 & $\mathrm{COOH}$ & $\mathrm{H}$ \\
\hline 6 & & 2-Me \\
\hline $6 a$ & & 2-Me \\
\hline 7 & $6 \mathrm{mmO}-$ & 2-Me \\
\hline $7 a$ & $6 \mathrm{mnO}-\mathrm{COOMe}$ & 2-Me \\
\hline
\end{tabular}

\section{Pharmacology}

The studied compounds can influence on cell viability. Macrophages, as phagocytes during activation, are dynamic cells that characterize movement of cell membrane. To preliminary study of cytotoxic effect of tested compounds during experiments it was used method based on measurements of LDH leakage from the cells. Neither of the tested xanthone derivatives at concentration $50 \mu \mathrm{M}$ showed toxicity against J7774A.1 cells cultured for $24 \mathrm{~h}$, but at concentration $100 \mu \mathrm{M}$ most of them showed weak toxicity (Tab. 2). 
Tab. 2. Effects of tested compounds on cell viability (cytotoxic effect) determined with LDH method

\begin{tabular}{lc}
\hline Cpds & $\begin{array}{c}\text { Cytotoxic effect at } \\
\text { concentration 100 } \mu \mathrm{M}[\%] \\
\text { Mean } \pm \text { SEM }\end{array}$ \\
\hline Control & 0 \\
FAA & 0 \\
XAA & $5.0 \pm 1.0$ \\
1 & $1.0 \pm 0.6$ \\
2 & $1.2 \pm 0.7$ \\
3 & $3.9 \pm 0.8$ \\
4 & $8.2 \pm 2.6$ \\
5 & $7.5 \pm 1.8$ \\
6 & $3.9 \pm 1.0$ \\
7 & $6.3 \pm 1.5$ \\
\hline Percentages of cytotoxicity at concentration \\
$50 \mu$ of tested compounds were equal zero. \\
The cells were cultured with compounds for $24 \mathrm{~h}$. \\
The data are the mean values from three \\
experiments.
\end{tabular}

Stimulation of macrophages by PMA induces process, named respiratory burst, mainly combined with generation of reactive forms of oxygen. The process remember phagocytosis when the cells destroying microorganisms. The phagocytosis is important in defense system against invading microbial pathogens. On the other hand overproduction of the oxygen metabolites may induce pathologies [36-38].

Tab. 3. Effects of tested compounds on chemiluminescence and generation of nitrite

\begin{tabular}{|c|c|c|c|c|c|c|}
\hline Cpds & $\begin{array}{l}\text { Chemiluminescence } \\
\text { [\% control] } \\
\text { Mean } \pm \text { SEM }\end{array}$ & $P<$ & $\begin{array}{l}\text { Nitrite } \\
\text { concentration } \\
{[\mu \mathrm{M}]} \\
\text { Mean } \pm \text { SEM }\end{array}$ & $P<$ & 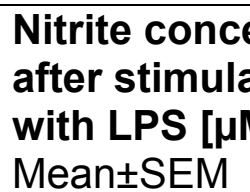 & $\begin{array}{l}\text { ntration } \\
\text { tion } \\
\text { M] } \\
P<\end{array}$ \\
\hline Control & 100 & & $5.7 \pm 1.0$ & & $25.7 \pm 0.4$ & \\
\hline FAA & $137 \pm 8.7$ & $0.05^{a}$ & $5.7 \pm 0.9$ & n.s. & $19.2 \pm 1.4$ & 0.05 \\
\hline XAA & $127 \pm 8.1$ & 0.05 & $5.8 \pm 0.8$ & n.s & $21.4 \pm 1.4$ & n.s. \\
\hline 1 & $149 \pm 12.7$ & 0.05 & $5.5 \pm 0.5$ & n.s & $21.8 \pm 0.6$ & 0.01 \\
\hline 2 & $112 \pm 4.6$ & n.s. & $5.8 \pm 0.2$ & n.s & $20.3 \pm 0.9$ & 0.01 \\
\hline 3 & $114 \pm 13.3$ & n.s. & $7.2 \pm 0.7$ & n.s & $23.1 \pm 1.0$ & n.s. \\
\hline 4 & $113 \pm 2.3$ & 0.02 & $7.2 \pm 0.9$ & n.s & $24.3 \pm 1.5$ & n.s. \\
\hline 5 & $72 \pm 6.9$ & 0.05 & $7.3 \pm 0.9$ & n.s & $22.8 \pm 1.8$ & n.s. \\
\hline 6 & $117 \pm 5.8$ & n.s. & $7.2 \pm 0.5$ & n.s & $21.9 \pm 1.3$ & n.s. \\
\hline 7 & $131 \pm 4.6$ & 0.005 & $5.5 \pm 0.4$ & n.s & $22.3 \pm 1.2$ & n.s. \\
\hline
\end{tabular}


Chemiluminescence (Tab. 3) was only inhibited in the presence of compound $\mathbf{5}$ with the 2-thio-2-methyl propionic moiety. Other of tested compounds increased or did not change chemiluminescence. In study [12] FAA and xanthnone acetic analogues these compounds changed PMA stimulated generation of superoxide anion by murine macrophages dependent on concentration.

Compounds at lower concentration stimulated but at higher concentration decreased generation of superoxide anion. Early studies with hydroxy and methoxyxanthone derivatives [28] showed stimulation of protein kinase $C$ (PKC) isoforms. PMA induces respiratory burst via PKC [29]. This mechanism may be in the presence of tested compounds. Compounds XAA and 1 (different position of the methyl carboxyl group) showed similar activity. Stimulation the J-774A.1 cells, as a model cells, by LPS induces generation of nitric oxide radical. The strong stimulation of macrophages in the body by pathogens generating a big amount of nitric oxygen and many other reactive substances destroying an environmental of the body. The activity of nitric oxide is dependent on place and concentration and this process is very difficult to control [39-41]. Tested compounds did not change generation of nitrite by unstimulated cells and slightly decreased generation of nitrite by stimulated J-774A.1 cells with LPS. The most activity in decreasing of nitrite generation was observed in the presence of FAA and xanthone derivatives with methyl carboxyl group. This result is in opposition to data by Thomsen [30]. That study was performed with FAA at higher concentration. Only 5,6-dimethyl-XAA was more active at low concentration. In our study concentration of tested compounds was lower because of cytotoxic effect.

The tested compounds in our study have not hydroxyl substituents at the main xanthone structure. The hydroxyxanthone derivatives, tested by other authors, showed an inhibitory effect on stimulated macrophages [31,32]. It is probably dependent on an activity of these groups to reaction with oxygen and nitrogen intermediates.

The chosen most active xanthone derivatives, after additional studies, may be use as a modulator of process accompanying with reactions of free radicals.

Further studies on the biological effects of the active xanthone derivatives are in progress.

\section{Experimental}

\section{Chemistry}

${ }^{1} \mathrm{H}$ NMR and ${ }^{13} \mathrm{C}$ NMR spectra were recorded on a Bruker spectrometer $500.13 \mathrm{MHz}$, using signal from DMSO in DMSO- $\mathrm{d}_{6}$ and $\mathrm{TMS}$ in $\mathrm{CDCl}_{3}$ as internal standards. MS were recorded using an AMD-604 mass spectrometer (70eV). The IR spectra were recorded on a Perkin Elmer or Jasco FT/IR 410 spectrometer (KBr pellets). M.p.s are uncorrected and were determined using a Büchi SMP-20 apparatus. Microanalyses were performed in the Department of Pharmaceutical Chemistry of Jagiellonian University, Medical College. All the results were within an acceptable range.

Mps ( $\left.{ }^{\circ} \mathrm{C}\right)$ for: XAA: 207-209 (214-215 [7], 205-207 [21]); 1: 215-217 (224-226 [7], 217219 [21], 215-217 [22]; 2: 236-238 [22]; 3: 222-224 [22]; 4: 182-184 [22]; 5: 205--206 [23]. 
Methyl (9-oxo-9H-xanthen-4-yl)acetate (XAAa)

Yield: $76 \%$, m.p. $155-157^{\circ} \mathrm{C}$ (methanol). IR (cm $\left.{ }^{-1}\right): 2950,1732,1655,1616,1603,1446$, 1347, 1226, 1215, 1172. ${ }^{1} \mathrm{H}$ NMR $(500.13 \mathrm{MHz})\left(\delta, \mathrm{ppm}, \mathrm{DMSO}-\mathrm{d}_{6}\right): 8.21(1 \mathrm{H}, \mathrm{dd}, \mathrm{J}=7.9$ $\mathrm{Hz}, \mathrm{J}=1.7 \mathrm{~Hz}, \mathrm{H}-8), 8.14(1 \mathrm{H}, \mathrm{dd}, \mathrm{J}=7.9 \mathrm{~Hz}, \mathrm{~J}=1.7 \mathrm{~Hz}, \mathrm{H}-1), 7.90(1 \mathrm{H}, \mathrm{ddd}, \mathrm{J}=8.5 \mathrm{~Hz}$, $\mathrm{J}=7.1 \mathrm{~Hz}, \mathrm{~J}=1.7 \mathrm{~Hz}, \mathrm{H}-6), 7.84(1 \mathrm{H}, \mathrm{dd}, \mathrm{J}=7.2 \mathrm{~Hz}, \mathrm{~J}=1.7 \mathrm{~Hz}, \mathrm{H}-3), 7.63(1 \mathrm{H}, \mathrm{dd}, \mathrm{J}=$ $8.5 \mathrm{~Hz}, \mathrm{~J}=1.0 \mathrm{~Hz}, \mathrm{H}-5), 7.51(1 \mathrm{H}, \mathrm{ddd}, \mathrm{J}=7.9 \mathrm{~Hz}, \mathrm{~J}=7.1 \mathrm{~Hz}, \mathrm{~J}=1.0 \mathrm{~Hz}, \mathrm{H}-7), 7.46(1 \mathrm{H}$, $\mathrm{dd}, \mathrm{J}=7.9 \mathrm{~Hz}, \mathrm{~J}=7.2 \mathrm{~Hz}, \mathrm{H}-2), 4.09\left(2 \mathrm{H}, \mathrm{s}, \mathrm{CH}_{2}\right), 3.69\left(3 \mathrm{H}, \mathrm{s}, \mathrm{CH}_{3}\right) .{ }^{13} \mathrm{C} \mathrm{NMR}(500.13$ $\mathrm{MHz})\left(\delta, \mathrm{ppm}, \mathrm{DMSO}-\mathrm{d}_{6}\right): 175.92(\mathrm{C}=\mathrm{O}), 170.85$ (COO), 155.17 (C-4b), 153.76 (C-4a), 136.79 (C-3), 135.56 (C-6), 125.90 (C-8), 124.96 (C-1), 124.51 (C-7), 124.33 (C-4), 123.83 (C-2), 121.02 (C-8b), 120.81 (C-8a), 118.10 (C-5), $51.86\left(\mathrm{OCH}_{3}\right), 34.49\left(\operatorname{Ar}-\mathrm{CH}_{2}\right)$. $\mathrm{C}_{16} \mathrm{H}_{12} \mathrm{O}_{4}(268.3)$.

\section{Methyl (6-methoxy-9-oxo-9H-xanthen-2-yl)acetate (2a)}

Yield: $73 \%$, m.p. $159-160^{\circ} \mathrm{C}$ (methanol). IR $\left(\mathrm{cm}^{-1}\right): 2950,1734,1654,1619,1595,1453$, 1256, 1162. ${ }^{1} \mathrm{H}$ NMR $(500.13 \mathrm{MHz})\left(\delta, \mathrm{ppm}\right.$, DMSO-d $\left.\mathrm{d}_{6}\right): 8.09(1 \mathrm{H}, \mathrm{d}, \mathrm{J}=8.8 \mathrm{~Hz}, \mathrm{H}-8), 8.07$ $(1 \mathrm{H}, \mathrm{dd}, \mathrm{J}=2.3 \mathrm{~Hz}, \mathrm{~J}=0.5 \mathrm{~Hz}, \mathrm{H}-1), 7.74(1 \mathrm{H}, \mathrm{dd}, \mathrm{J}=8.5 \mathrm{~Hz}, \mathrm{~J}=2.3 \mathrm{~Hz}, \mathrm{H}-3), 7.58(1 \mathrm{H}$, $\mathrm{dd}, \mathrm{J}=8.5 \mathrm{~Hz}, \mathrm{~J}=0.5 \mathrm{~Hz}, \mathrm{H}-4), 7.14(1 \mathrm{H}, \mathrm{d}, \mathrm{J}=2.4 \mathrm{~Hz}, \mathrm{H}-5), 7.04(1 \mathrm{H}, \mathrm{dd}, \mathrm{J}=8.8 \mathrm{~Hz}, \mathrm{~J}=$ $2.4 \mathrm{~Hz}, \mathrm{H}-7), 3.93\left(3 \mathrm{H}, \mathrm{s}, \mathrm{CH}_{3} \mathrm{OAr}\right), 3.89\left(2 \mathrm{H}, \mathrm{s}, \mathrm{CH}_{2}\right), 3.65\left(3 \mathrm{H}, \mathrm{s}, \mathrm{CH}_{3}\right) .{ }^{13} \mathrm{C}$ NMR $(500.13$

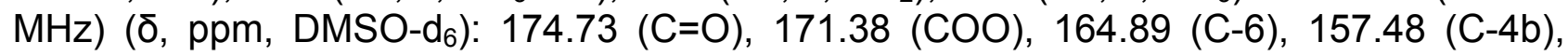
154.55 (C-4a), 136.21 (C-3), 130.58 (C-2), 127.51 (C-8), 126.27 (C-1), 120.87 (C-8b), 117.82 (C-4), 114.84 (C-8a),113.54 (C-7), 100.52 (C-5), $56.07\left(\mathrm{CH}_{3} \mathrm{OAr}\right), 51.71\left(\mathrm{OCH}_{3}\right)$, $39.05\left(\mathrm{CH}_{2}\right) \cdot \mathrm{C}_{17} \mathrm{H}_{14} \mathrm{O}_{5}(298.3)$.

\section{( \pm )-2-[(7-Methyl-9-oxo-9H-xanthen-3-yl)oxy]propanoic acid (6)}

Yield: $63 \%$, m.p. $201-203^{\circ} \mathrm{C}$ (ethanol). IR $\left(\mathrm{cm}^{-1}\right): 3438,2922,1749,1655,1618,1578$, 1481, 1310, 1257, 1234, 1206, 1177, 1113. ${ }^{1} \mathrm{H}$ NMR $(500.13 \mathrm{MHz})\left(\delta, \mathrm{ppm}, \mathrm{DMSO}-\mathrm{d}_{6}\right)$ : $13.30\left(1 \mathrm{H}\right.$, bs, $\left.\mathrm{CO}_{2} \mathrm{H}\right), 8.10(1 \mathrm{H}, \mathrm{dd}, \mathrm{J}=8.7 \mathrm{~Hz}, \mathrm{~J}=0.5 \mathrm{~Hz}, \mathrm{H}-8), 7.94(1 \mathrm{H}, \mathrm{ddd}, \mathrm{J}=2.3$ $\mathrm{Hz}, \mathrm{J}=0.9 \mathrm{~Hz}, \mathrm{~J}=0.5 \mathrm{~Hz}, \mathrm{H}-1), 7.65(1 \mathrm{H}, \mathrm{ddd}, \mathrm{J}=8.5 \mathrm{~Hz}, \mathrm{~J}=2.3 \mathrm{~Hz}, \mathrm{~J}=0.6 \mathrm{~Hz}, \mathrm{H}-3)$, $7.53(1 \mathrm{H}, \mathrm{ddd}, \mathrm{J}=8.5 \mathrm{~Hz}, \mathrm{~J}=0.5 \mathrm{~Hz}, \mathrm{~J}=0.5 \mathrm{~Hz}, \mathrm{H}-4), 7.03(1 \mathrm{H}, \mathrm{dd}, \mathrm{J}=8.7 \mathrm{~Hz}, \mathrm{~J}=2.3$ $\mathrm{Hz}, \mathrm{H}-7), 7.01(1 \mathrm{H}, \mathrm{dd}, \mathrm{J}=2.3 \mathrm{~Hz}, \mathrm{~J}=0.5 \mathrm{~Hz}, \mathrm{H}-5), 5.13(1 \mathrm{H}, \mathrm{q}, \mathrm{J}=6.8 \mathrm{~Hz}, \mathrm{CH}), 2.43(3 \mathrm{H}$, ddd, J = 0.9 Hz, J = 0.6 Hz, J = 0.5 Hz, CH $\left.{ }_{3} \mathrm{Ar}\right), 1.58\left(3 \mathrm{H}, \mathrm{d}, \mathrm{J}=6.8 \mathrm{~Hz}, \mathrm{CH}_{3} \mathrm{R}\right) .{ }^{13} \mathrm{C} \mathrm{NMR}$ $(500.13 \mathrm{MHz})\left(\delta, \mathrm{ppm}, \mathrm{DMSO}-\mathrm{d}_{6}\right): 174.77(\mathrm{C}=\mathrm{O}), 172.16(\mathrm{COOH}), 162.91(\mathrm{C}-6), 157.17$ (C-4b), 153.79 (C-4a), 135.98 (C-3), 133.66 (C-2), 127.64 (C-8), 125.10 (C-1), 120.78 (C8b), 117.67 (C-4), 115.19 (C-8a), 113.71 (C-7), 101.46 (C-5), 72.15 (CH-O-Ar), $20.25\left(\mathrm{CH}_{3}-\right.$ $\mathrm{Ar}), 17.91\left(\mathrm{CH}_{3}-\mathrm{CH}\right)$. MS (m/z): $298\left(\mathrm{M}^{+}\right), 253$ (100\%), 226, 209, 197, 181, 169, 153, 126, 115. $\mathrm{C}_{17} \mathrm{H}_{14} \mathrm{O}_{5}$ (298.3).

\section{(士)-Ethyl 2-[(7-methyl-9-oxo-9H-xanthen-3-yl)oxy]propanoate (6a)}

Yield: $71 \%$, m.p. $121-123^{\circ} \mathrm{C}$ (ethanol). IR ( $\left.\mathrm{cm}^{-1}\right)$ : 2991, 2932, 1732, 1656, 1614, 1591, $1480,1444,1305,1289,1252,1232,1175,1107 .{ }^{1} \mathrm{H}$ NMR $(500.13 \mathrm{MHz})(\delta, \mathrm{ppm}$, DMSO$\left.\mathrm{d}_{6}\right): 8.10(1 \mathrm{H}, \mathrm{dd}, \mathrm{J}=8.7 \mathrm{~Hz}, \mathrm{~J}=0.5 \mathrm{~Hz}, \mathrm{H}-8), 7.95(1 \mathrm{H}, \mathrm{ddd}, \mathrm{J}=2.3 \mathrm{~Hz}, \mathrm{~J}=0.9 \mathrm{~Hz}, \mathrm{~J}=$ $0.5 \mathrm{~Hz}, \mathrm{H}-1), 7.66(1 \mathrm{H}, \mathrm{ddd}, \mathrm{J}=8.6 \mathrm{~Hz}, \mathrm{~J}=2.3 \mathrm{~Hz}, \mathrm{~J}=0.6 \mathrm{~Hz}, \mathrm{H}-3), 7.53(1 \mathrm{H}, \mathrm{dd}, \mathrm{J}=8.6$ $\mathrm{Hz}, \mathrm{J}=0.5 \mathrm{~Hz}, \mathrm{H}-4), 7.06(1 \mathrm{H}, \mathrm{dd}, \mathrm{J}=2.4 \mathrm{~Hz}, \mathrm{~J}=0.5 \mathrm{~Hz}, \mathrm{H}-5), 7.04(1 \mathrm{H}, \mathrm{dd}, \mathrm{J}=8.7 \mathrm{~Hz}, \mathrm{~J}$ $=2.4 \mathrm{~Hz}, \mathrm{H}-7), 5.28(1 \mathrm{H}, \mathrm{q}, \mathrm{J}=6.8 \mathrm{~Hz}, \mathrm{CH}), 4.19(2 \mathrm{H}, \mathrm{dq}, \mathrm{J}=10.9 \mathrm{~Hz}, \mathrm{~J}=7.1 \mathrm{~Hz}$, $\mathrm{C} \underline{H} \mathrm{H}(\mathrm{Et})), 4.17(2 \mathrm{H}, \mathrm{dq}, \mathrm{J}=10.9 \mathrm{~Hz}, \mathrm{~J}=7.1 \mathrm{~Hz}, \mathrm{CH} \underline{\mathrm{H}}(\mathrm{Et})), 2.44(3 \mathrm{H}, \mathrm{dd}, \mathrm{J}=0.9 \mathrm{~Hz}, \mathrm{~J}=$ $\left.0.6 \mathrm{~Hz}, \mathrm{CH}_{3} \mathrm{Ar}\right), 1.59\left(3 \mathrm{H}, \mathrm{d}, \mathrm{J}=6.8 \mathrm{~Hz}, \mathrm{CH}_{3}\right), 1.20\left(3 \mathrm{H}, \mathrm{t}, \mathrm{J}=7.1 \mathrm{~Hz}, \mathrm{CH}_{3}(\mathrm{Et})\right) .{ }^{13} \mathrm{C} \mathrm{NMR}$ $(500.13 \mathrm{MHz})\left(\delta, \mathrm{ppm}, \mathrm{DMSO}-\mathrm{d}_{6}\right): 174.79(\mathrm{C}=\mathrm{O}), 170.58(\mathrm{COOH}), 162.67(\mathrm{C}-6), 157.18$ 
(C-4b), 153.81 (C-4a),136.05 (C-3), 133.71 (C-2), 127.72 (C-8), 125.12 (C-1), 120.79 (C8b), 117.69 (C-4), 115.37 (C-8a), 113.68 (C-7), 101.70 (C-5), 72.17 (CH-O-Ar), $61.02\left(\mathrm{CH}_{2}\right.$ (Et)), $20.25\left(\mathrm{CH}_{3}-\mathrm{Ar}\right), 17.87\left(\mathrm{CH}_{3}-\mathrm{CH}\right), 13.89\left(\mathrm{CH}_{3}(\mathrm{Et})\right) . \mathrm{C}_{19} \mathrm{H}_{18} \mathrm{O}_{5}(326.3)$.

\section{2-Methyl-2-[(7-methyl-9-oxo-9H-xanthen-3-yl)oxy]propanoic acid (7)}

Yield: $62 \%$, m.p. $212-214^{\circ} \mathrm{C}$ (ethanol). IR $\left(\mathrm{cm}^{-1}\right)$ : 3436, 2941, 1743, 1654, 1603, 1575, 1448, 1250, 1140, 1108. ${ }^{1} \mathrm{H}$ NMR $(500.13 \mathrm{MHz})\left(\delta, \mathrm{ppm}, \mathrm{DMSO}-\mathrm{d}_{6}\right): 13.40\left(1 \mathrm{H}, \mathrm{s}, \mathrm{CO}_{2} \mathrm{H}\right)$, $8.09(1 \mathrm{H}, \mathrm{d}, \mathrm{J}=8.9 \mathrm{~Hz}, \mathrm{H}-8), 7.92(1 \mathrm{H}, . \mathrm{d}, \mathrm{J}=2.3 \mathrm{~Hz}, \mathrm{H}-1), 7.61(1 \mathrm{H}, \mathrm{ddd}, \mathrm{J}=8.4 \mathrm{~Hz}, \mathrm{~J}=$ $2.3 \mathrm{~Hz}, \mathrm{~J}=0.6 \mathrm{~Hz}, \mathrm{H}-3), 7.50(1 \mathrm{H}, \mathrm{d}, \mathrm{J}=8.5 \mathrm{~Hz}, \mathrm{H}-4), 6.95(1 \mathrm{H}, \mathrm{dd}, \mathrm{J}=8.9 \mathrm{~Hz}, \mathrm{~J}=2.4 \mathrm{~Hz}$, $\mathrm{H}-7), 6.82(1 \mathrm{H}, \mathrm{d}, \mathrm{J}=2.4 \mathrm{~Hz}, \mathrm{H}-5), 2.42\left(3 \mathrm{H}, \mathrm{s}, \mathrm{CH}_{3} \mathrm{Ar}\right), 1.67\left(6 \mathrm{H}, \mathrm{s}, 2 \mathrm{xCH}_{3}\right) .{ }^{13} \mathrm{C} \mathrm{NMR}$ $(500.13 \mathrm{MHz})\left(\delta, \mathrm{ppm}, \mathrm{DMSO}_{-} \mathrm{d}_{6}\right): 175.99(\mathrm{CO}), 175.36(\mathrm{COOH}), 162.16(\mathrm{C}-6), 157.83(\mathrm{C}-$ 4b), 154.82 (C-4a), 137.11 (C-3), 134.77 (C-2), 128.60 (C-8), 126.11 (C-1), 121.73 (C-8b), 118.75 (C-4), 116.62 (C-7), 116.18 (C-8a), 104.74 (C-5), 80.73 (C-O-Ar), 26.03 (2x $\left.\mathrm{CH}_{3}\right)$, $21.34\left(\mathrm{CH}_{3}-\mathrm{Ar}\right) . \mathrm{MS}$ (m/z): $312\left(\mathrm{M}^{+}\right), 267,226(100 \%), 197,181,169,115 . \mathrm{C}_{18} \mathrm{H}_{16} \mathrm{O}_{5}$ (312.3).

\section{Methyl 2-methyl-2-[(7-methyl-9-oxo-9H-xanthen-3-yl)oxy]propanoate (7a)}

Yield: $74 \%$, m.p. $120-122^{\circ} \mathrm{C}$ (methanol). IR (cm $\left.{ }^{-1}\right)$ : 2993, 2956, 1737, 1651, 1620, 1593, 1482, 1445, 1301, 1261, 1172. ${ }^{1} \mathrm{H}$ NMR $(500.13 \mathrm{MHz})\left(\delta, \mathrm{ppm}, \mathrm{DMSO}-\mathrm{d}_{6}\right): 8.09(1 \mathrm{H}, \mathrm{d}, \mathrm{J}=$ $8.9 \mathrm{~Hz}, \mathrm{H}-8), 7.93(1 \mathrm{H}, . \mathrm{d}, \mathrm{J}=2.3 \mathrm{~Hz}, \mathrm{H}-1), 7.63(1 \mathrm{H}, \mathrm{ddd}, \mathrm{J}=8.5 \mathrm{~Hz}, \mathrm{~J}=2.3 \mathrm{~Hz}, \mathrm{~J}=0.7$ $\mathrm{Hz}, \mathrm{H}-3), 7.50(1 \mathrm{H}, \mathrm{d}, \mathrm{J}=8.5 \mathrm{~Hz}, \mathrm{H}-4), 6.91(1 \mathrm{H}, \mathrm{dd}, \mathrm{J}=8.8 \mathrm{~Hz}, \mathrm{~J}=2.4 \mathrm{~Hz}, \mathrm{H}-7), 6.81$ $(1 \mathrm{H}, \mathrm{d}, \mathrm{J}=2.4 \mathrm{~Hz}, \mathrm{H}-5), 3.76\left(3 \mathrm{H}, \mathrm{s}, \mathrm{CH}_{3} \mathrm{O}\right), 2.43\left(3 \mathrm{H}, \mathrm{s}, \mathrm{CH}_{3} \mathrm{Ar}\right), 1.68\left(6 \mathrm{H}, \mathrm{s}, 2 \times \mathrm{CH}_{3}\right) .{ }^{13} \mathrm{C}$ NMR (500.13 MHz) (ס, ppm, DMSO-d 6 ): 176.01 (CO), $174.10(\mathrm{COOH}), 161.79(\mathrm{C}-6)$, 157.84 (C-4b), 154.86 (C-4a), 137.24 (C-3), 134.84 (C-2), 128.73 (C-8), 126.13 (C-1), 121.73 (C-8b), 118.82 (C-4), 116.55 (C-7), 116.48 (C-8a), 105.27 (C-5), 80.75 (C-O-Ar), $58.81\left(\mathrm{OCH}_{3}\right), 26.00\left(2 \times \mathrm{CH}_{3}\right), 21.31\left(\mathrm{CH}_{3}-\mathrm{Ar}\right)$. MS (m/z): $312\left(\mathrm{M}^{+}\right)$, 267, 226 (100\%), 197, 181, 169, 115. $\mathrm{C}_{19} \mathrm{H}_{18} \mathrm{O}_{5}$ (326.3).

\section{Pharmacology}

Compounds were dissolved in RPMI 1640 medium without phenol red (Gibco, Grand Island, NY) or in Hanks buffer (HBSS) pH 7.4 without phenol red (Gibco, Grand Island, NY).

\section{J-774A.1 cells}

Murine J-774A.1 macrophages were obtained from German Collection of Microorganisms and Cell Cultures, Dept. Human and Animal Cell Cultures in Braunschweig. During the study cells were grown in RPMI 1640 medium without phenol red (Gibco, Grand Island, NY) supplemented with $10 \%$ fetal calf serum (FCS) (South American Origin, BIO WHITTAKER EUROPE, Verviers, Belgium), penicillin (100 U/mL) and streptomycin (100 $\mu \mathrm{g} / \mathrm{mL}$ ) at $37^{\circ} \mathrm{C}$ and $5 \% \mathrm{CO}_{2}$ atmosphere.

During culture and experiments cell viability was controlled by lactate dehydrogenase method (Cytotoxicity Detection Kit (LDH)-Roche Diagnostics GmbH, Mannheim, Germany).

\section{Cell stimulation}

The adherent cells were incubated with tested compounds $(0.5 \mathrm{~h})$ and next stimulated with 
lipopolisaccharide (100 ng/mL) from E.coli (0127:B8) (Calbiochem, La Jolla, CA, USA) After $24 \mathrm{~h}$ culture of the cells the supernatants were removed and assayed for levels of nitrite.

\section{Nitrite assay}

Nitrite concentration in the culture medium was measured by a microplate assay method, based on the Griess reaction [33]. Equal volumes of culture medium supernatant and Griess reagent $(0.5 \%$ sulfanilamide, $0.05 \%$ naphthylene-diamide dihydrochloride in $2.5 \%$ $\mathrm{H}_{3} \mathrm{PO}_{4}$ ) were added to microplate and incubated at $25^{\circ} \mathrm{C}$ for $10 \mathrm{~min}$. The absorbance of culture medium and Griess reagent at 550nm was determined with Microplate Reader ELX 800 (Bio-tek Instruments, Inc., Winooski, VT, USA).

\section{Chemiluminescence}

Into $10^{6}$ cells with tested compounds or with HBSS (controls), luminol (Sigma, St. Louis, $\mathrm{MO}$, USA) solution was added, giving a final concentration $0.1 \mathrm{mM}$ and 5 min later phorbol myristate acetate (PMA) (Sigma, St. Louis, MO, USA) solution (final concentration $0.8 \mu \mathrm{M}$ ) as stimulus was used. The final volume of each sample was $1 \mathrm{~mL}$. Chemiluminescence (CL) was measured for $20 \mathrm{~min}$ (5 min with luminol and $15 \mathrm{~min}$, after that, PMA was added) using a system equipped with a photomultiplier 9514s from THORN EMI (Middlesex, England) [34, 35]. The intensity of CL was determined by measuring counts/min and by calculating the area under the CL curve. Then the percentages of control were calculated.

\section{Authors' Statement}

\section{Competing Interests}

The authors declare no conflict of interest.

\section{References}

[1] Lin CN, Liou SJ, Lee TH, Chuang YC, Won SJ.

Xanthone derivatives as potential anti-cancer drugs.

J Pharm Pharmacol. 1996; 48: 539-544.

PMid:8799883

[2] Kinghorn AD, Farnsworth NR, Soejarto DD, Cordell GA, Pezzuto JM, Udeani GO, Wani MC, Wall ME, Navarro HA, Kramer RA, Menendez AT, Fairchild CR, Lane KE, Forenza S, Vyas DM, Lam KS,

Shu YZ.

Novel strategies for the discovery of plant-derived anticancer agents.

Pure Appl Chem. 1999; 71: 1611-1618.

doi:10.1351/pac199971091611

[3] Atassi G, Briet P, Berthelon JJ, Collonges F.

Synthesis and antitumor activity of some 8-substituted 4-oxo-4H-1-benzopyrans.

Eur J Med Chem. 1985; 20: 393-402.

[4] Smith GP, Calveley SB, Smith MJ, Baguley BC.

Flavone acetic acid (NSC 347512) induces haemorrhagic necrosis of mouse colon 26 and 38 tumours. Eur J Cancer Clin Oncol. 1987; 23: 1209-1211. doi:10.1016/0277-5379(87)90157-X 
[5] Capolongo LS, Balconi G, Ubezio P, Giavazzi R, Taraboletti G, Regonesi A, Yoder OC, D'Incalci M. Antiproliferative properties of flavone acetic acid (NSC 347512) (LM 975), a new anticancer agent. Eur J Cancer Clin Oncol. 1987; 23: 1529-1535. doi:10.1016/0277-5379(87)90096-4

[6] Aitken RA, Bibby MC, Double JA, Phillips RM, Sharma SK.

Synthesis and antitumour activity of new derivatives of flavone-8-acetic acid (FAA). Part 1: 6-Methyl derivatives.

Arch Pharm. 1996; 329: 489-497.

doi:10.1002/ardp.19963291104

[7] Rewcastle GW, Atwell GJ, Baguley BC, Calveley SB, Denny WA.

Potential antitumor agents. 58. Synthesis and structure-activity relationships of substituted

xanthenone-4-acetic acids active against the colon 38 tumor in vivo.

J Med Chem. 1989; 32: 793-799.

doi:10.1021/jm00124a012

[8] Rewcastle GW, Atwell GJ, Li ZA, Baguley BC, Denny WA.

Potential antitumor agents. 61. Structure-activity relationships for in vivo colon 38 activity among

disubstituted 9-oxo-9H-xanthene-4-acetic acids.

J Med Chem. 1991; 34: 217-222.

doi:10.1021/jm00105a034

[9] Zhou S, Kestell P, Baguley BC, Paxton JW.

Preclinical factors influencing the relative contributions of phase I and II enzymes to the metabolism of the experimental anti-cancer drug 5,6-dimethylxanthenone-4-acetic acid.

Biochem Pharmacol. 2003; 65: 109-120.

doi:10.1016/S0006-2952(02)01425-9

[10] Gobbi S, Rampa A, Bisi A, Belluti F, Valenti P, Caputo A, Zampiron A, Carrara M.

Synthesis and antitumor activity of new derivatives of xanthen-9-one-4-acetic acid.

J Med Chem. 2002; 45: 4931-4939.

doi:10.1021/jm020929p

[11] Wiltrout RH, Hornung RL.

Natural products as antitumor agents: direct versus indirect mechanisms of activity of flavonoids.

J Natl Cancer Inst. 1988; 80: 220-222.

doi:10.1093/jnci/80.4.220

[12] Thomsen LL, Baguley BC, Ching LM, Gavin JB.

Modulation of superoxide production from murine macrophages by the antitumor agent flavone acetic acid and xanthenone acetic acid analogues.

Biochem Pharmacol. 1992; 43: 386-389.

doi:10.1016/0006-2952(92)90304-2

[13] Hodnick WF, Kung FS, Roettger WJ, Bohmont CW, Pardini RS.

Inhibition of mitochondrial respiration and production of toxic oxygen radicals by flavonoids. A

structure-activity study.

Biochem Pharmacol. 1986; 35: 2345-2357.

doi:10.1016/0006-2952(86)90461-2

[14] Ching LM, Baguley BC.

Hyporesponsiveness of macrophages from $\mathrm{C} 3 \mathrm{H} / \mathrm{HeJ}$ (endotoxin-resistant) mice to the antitumour agent flavone acetic acid (NSC 347512).

Eur J Cancer Clin Oncol. 1989; 25: 1513-1515.

doi:10.1016/0277-5379(89)90116-8

[15] Thomsen LL, Ching LM, Joseph WR, Baguley BC, Gavin JB.

Nitric oxide production in endotoxin-resistant $\mathrm{C} 3 \mathrm{H} / \mathrm{HeJ}$ mice stimulated with flavone-8-acetic acid and xanthenone-4-acetic acid analogues.

Biochem Pharmacol. 1992, 43: 2401-2406.

doi:10.1016/0006-2952(92)90319-E 
[16] Stuehr DJ, Nathan CF.

Nitric oxide. A macrophage product responsible for cytostasis and respiratory inhibition in tumor target cells.

J Exp Med. 1989; 169: 1543-1555.

doi:10.1084/jem.169.5.1543

[17] Klebanoff SJ.

Reactive nitrogen intermediates and antimicrobial activity: role of nitrite.

Free Radic Biol Med. 1993; 14: 351-360.

doi:10.1016/0891-5849(93)90084-8

[18] Król W, Czuba ZP, Threadgill MD, Cunningham BDM, Pietsz G.

Inhibition of nitric oxide (NO.) production in murine macrophages by flavones.

Biochem Pharmacol. 1995; 50: 1031-1035.

doi:10.1016/0006-2952(95)00237-T

[19] Tosk J, Lau BHS, Lui P, Myers RC, Torrey RR.

Chemiluminescence in a macrophage cell line modulated by biological response modifiers.

J Leukocyte Biol. 1989; 46: 103-108.

PMid:2746137

[20] Raso GM, Meli R, Di Carlo G, Pacilio M, Di Carlo R.

Inhibition of inducible nitric oxide synthase and cyclooxygenase-2 expression by flavonoids in macrophage J774A.1.

Life Sci. 2001; 68: 921-931.

doi:10.1016/S0024-3205(00)00999-1

[21] Livingstone R, editor.

In: Coffey's. Rodd's Chemistry of Carbon Compounds.

2nd ed. Volume 4, Part E.

Amsterdam: Elsevier, 1977; 316-333.

[22] Marona H, Eckstein M, Krupińska J, Mazur J, Piotrowicz J, Cebo B.

Synthesis and some biological properties of 2-xanthonylalkyl-(alkoxy) carboxylic acids.

Pol J Pharmacol Pharm. 1986; 38: 107-114.

PMid:3489931

[23] Marona $\mathrm{H}$.

[Synthesis of some 2-xanthonylmethylthioalkanoic acids.]

Acta Pol Pharm. 1988; 45: 31-34

[24] Marona H, Pękala E, Filipek B, Maciag D, Szneler E.

Pharmacological properties of some aminoalkanolic derivatives of xanthone.

Pharmazie. 2001; 56: 567-572.

PMid:11487977

[25] Link G.

(DRP) 80986.

Chem Zbl. 1895; 11: 90.

[26] von Thiele A, Jahn U, Adrian RW.

[Beclobrate and eniclobrate hydrochloride, new diphenylmethane derivatives as agents for lowering cholesterol and triglyceride levels. Part I: Synthesis and consideration of structure-activity relationships.]

Arzneimittelforschung. 1979; 29: 5.

PMid:582967

[27] Gaion RM, Valenti P, Montanari P, Da Re P.

Xanthone analogues of clofibrate / Synthesis and biological evaluation as antagonists of lipolysis in vitro.

Arzneimittelforschung. 1982; 32: 499-502.

PMid:7201826 
[28] Saraiva L, Fresco P, Pinto E, Sousa E, Pinto M, Goncalves J.

Synthesis and in vivo modulatory activity of protein kinase $\mathrm{C}$ of xanthone derivatives.

Bioorg Med Chem. 2002; 10: 3219-3227.

doi:10.1016/S0968-0896(02)00169-4

[29] Gescher A, Dale IL.

Protein-kinase $\mathrm{C}$ - a novel target for rational anti-cancer drug design.

Anticancer Drug Design. 1989; 4: 93-105.

PMid:2679620

[30] Thomsen LL, Ching LM, Baguley BC.

Evidence for the production of nitric oxide by activated macrophages treated with the antitumor agents flavone-8-acetic acid and xanthenone-4-acetic acid.

Cancer Res. 1990; 50: 6966-6970.

PMid:/2170013

[31] Jeong GS, Lee DS, Kim YC.

Cudratricusxanthone A from Cudrania tricuspidata suppresses pro-inflammatory mediators through expression of anti-inflammatory heme oxygenase-1 in RAW264.7 macrophages.

Int Immunopharmacol. 2009; 9: 241-246.

doi:10.1016/j.intimp.2008.11.008

[32] Chen LG, Yang LL, Wang CC.

Anti-inflammatory activity of mangostins from Garcinia mangostana.

Food Chem Toxicol. 2008; 46: 688-693.

doi:10.1016/j.fct.2007.09.096

[33] Green LC, Wagner DA, Glogowski J, Skipper PI, Wishnok JS, Tannenbaum SR.

Analysis of nitrate, nitrite, and $\left({ }^{15} \mathrm{~N}\right)$ nitrate in biological fluids.

Anal Biochem. 1982; 126: 131-138.

doi:10.1016/0003-2697(82)90118-X

[34] Król W, Czuba Z, Scheller S, Gabryś J, Grabiec S, Shani J.

Anti-oxidant property of ethanolic extract of propolis (EEP) as evaluated by inhibiting the

chemiluminescence oxidation of luminol.

Biochem Int. 1990; 21: 593-597.

PMid:2241984

[35] Czuba ZP, Król W, Hasinski P, Nowowiejska A.

The effects of taxol (paclitaxel) on chemiluminescence of neutrophils, macrophages and J.774.2 cell line.

Acta Biochem Pol. 1998; 45: 103-106.

PMid:9701502

[36] Winterbourn CC, Kettle AJ.

Radical-radical reactions of superoxide: a potential route to toxicity.

Biochem Biophys Res Commun. 2003; 305; 729-736.

doi:10.1016/S0006-291X(03)00810-6

[37] Hultqvist M, Olsson LM, Gelderman KA, Holmdahl R.

The protective role of ROS in autoimmune disease.

Trends Immunol. 2009; 30: 201-208.

doi:10.1016/j.it.2009.03.004

[38] Greaves DR, Channon KM.

Inflammation and immune responses in atherosclerosis.

Trends Immunol. 2002; 23: 535-541.

doi:10.1016/S1471-4906(02)02331-1 
[39] Giang PM, Son PT, Jin HZ, Lee JH, Lee JJ.

Comparative study on inhibitory activity of Zerumbone and Zerumbone 2,3-Epoxide on NF-kB activation and $\mathrm{NO}$ production.

Sci Pharm. 2009; 77: 589-595.

doi:10.3797/scipharm.0907-16

[40] Lundberg JO, Weitzberg E, Gladwin MT.

The nitrate-nitrite-nitric oxide pathway in physiology and therapeutics.

Nat Rev Drug Discov. 2008; 7: 156-167.

doi:10.1038/nrd2466

[41] Padwad Y, Ganju L, Jain M, Chanda S, Karan D, Banerjee PK, SawhneyRC.

Effect of extract of Seabuckthom on lipopolisaccharide induced inflammatory response in murine macrophages.

Int Immunopharmacol. 2006; 6: 46-52.

doi:10.1016/j.intimp.2005.07.015 